\title{
Problema proposto ou problema resolvido: qual a diferença?*
}

Jader Otavio Dalto

Regina Luzia Corio de Buriasco

Universidade Estadual de Londrina

Correspondência:

Regina Luzia Corio de Buriasco

Rua Eduardo Benjamin Hosken, 173, apto 501

86020-440 - Londrina - PR

e-mail: reginaburiasco@hasner.com.br

\section{Resumo}

Este trabalho apresenta um estudo sobre a produção escrita presente na questão comum à $8^{\text {a }}$ série do ensino fundamental e à $3^{\text {a }}$ série do ensino médio da Prova de Questões Abertas de Matemática da Avaliação do Rendimento Escolar do Estado do Paraná - AVA/2002. A abordagem metodológica adotada é predominantemente qualitativa, orientando-se pelas técnicas da análise de conteúdo como ferramenta de compreensão e inferência da produção escrita dos alunos em uma amostra de 97 provas. Após a correção e a realização de agrupamentos, foram identificadas quatro categorias de resolução da questão. Em cada categoria, foram inferidos enunciados de problemas que os estudantes parecem ter compreendido e resolvido a partir da interpretação que fizeram do enunciado da questão proposta. Como resultados gerais, tem-se que: a) as estratégias utilizadas pelos estudantes para resolver a questão não diferem muito de uma série para outra; b) o desempenho dos estudantes da $3^{\text {a }}$ série do ensino médio é melhor que o desempenho dos estudantes da $8^{\text {a }}$ série do ensino fundamental; c) a maioria dos estudantes resolveu a questão utilizando operações aritméticas como adição, subtração, multiplicação e divisão, o que está sendo considerado aqui como uma estratégia aritmética. A análise aponta ainda indícios de que o baixo desempenho dos estudantes está mais associado à dificuldade de compreender o enunciado da questão, e que eles ‘dominam' parte do 'conteúdo matemático' necessário à resolução desta.

\section{Palavras-chave}

Educação Matemática - Avaliação da aprendizagem em Matemática Escolar - Produção escrita - Acerto e erro em Matemática. 


\section{Problem posed or problem solved: what is the difference?}

Jader Otavio Dalto

Regina Luzia Corio de Buriasco

Universidade Estadual de Londrina

Contact:

Regina Luzia Corio de Buriasco

Rua Eduardo B. Hosken, 173, apto 501

86020-440 - Londrina - PR

e-mail: reginaburiasco@hasner.com.br

\begin{abstract}
This work describes a study about the written production present in a question that is common to the exams of the 8th grade of the fundamental education and 3rd grade of secondary education in the Mathematics Open Questions Exam of the Assessment of School Performance of the State of Paraná - AVA/2002. The methodological approach adopted here is predominantly qualitative, guided by the techniques of content analysis as a tool of inference and understanding of the written production of students in a sample of 97 exams. After correction and grouping, four question solution categories were identified. For each category an inference was made of the question formulation that students seem to have understood and solved based on the interpretation they made of the question proposed. In broad terms, results were that: a) strategies employed by students to solve the questions did not vary significantly between the two grades analyzed; b) the performance of students of the 3rd grade of secondary education was better than that of students from the 8th grade of fundamental education; c) most students solved the question using arithmetic operations such as addition, subtraction, multiplication and division, which is being considered here as an arithmetic strategy. The analysis also indicated that poor student performance is more closely related to difficulties in understanding the formulation of the question, and that students "master" part of the "mathematical content" necessary for its solution.
\end{abstract}

\section{Keywords}

Mathematical education - Learning assessment in school mathematics - Written production - Right and wrong answers in mathematics.

\footnotetext{
This article is based on a thesis (Dalto, 2007), and on part of a research program whose object is the written production of students and teachers in the Mathematics Open Questions Exam (Prova de Questões Abertas de Matemática) - AVA/2002.
} 
Avaliações estaduais, nacionais e internacionais do rendimento vêm fazendo parte do cotidiano escolar há alguns anos. Nas primeiras edições do Sistema de Avaliação do Rendimento Escolar do Estado do Paraná - AVA -, as provas de matemática continham apenas questões de múltipla escolha. Entretanto, na edição de 2002, uma prova de questões abertas de matemática foi resolvida por um terço dos alunos da $4^{\text {a }}$ e $8^{a}$ séries do ensino fundamental - EF - e $3^{\text {a }}$ série do ensino médio - EM. Essa Prova ${ }^{1}$ é composta por três ou quatro questões abertas, também chamadas discursivas. Nesse tipo de questão, o aluno deve ler a questão, compreender e interpretar o enunciado, escolher uma estratégia ou um procedimento para resolver a questão, registrar seu raciocínio, seus cálculos ou o que for necessário para somente então apresentar a resposta.

De modo geral, não apenas os resultados da AVA, mas também resultados das demais avaliações como SAEB, PISA entre outras, revelam o baixo desempenho dos estudantes brasileiros, em especial no que se refere à matemática. Contudo, tais resultados não garantem respostas a perguntas tais como: que "Matemática” os alunos conhecem? Como eles utilizam esse conhecimento na resolução de problemas, apresentando ou não uma solução correta?

Diante disso, procurou-se, a partir de uma amostra da Prova aplicada em todo o estado do Paraná, analisar a produção escrita de alunos na questão comum à Prova da $8^{\text {a }}$ série do EF e à da $3^{\text {a }}$ série do EM.

\section{A avaliação e o processo de ensino e de aprendizagem}

A avaliação é uma prática complexa que, apesar de ser objeto de estudo de pesquisadores da área da Educação há muitos anos, ainda é considerada por muitas pessoas como sendo apenas um ato de medida. Tal concepção simplista vai de encontro com as reais funções da avaliação que são apresentadas por autores como Abrantes (1995), Hadji (2001), Esteban
(2002), Buriasco (2002; 2004). Em suas pesquisas, esses autores afirmam que a avaliação deve estar a serviço da ação pedagógica e deve ser também um mecanismo de regulação do processo educativo.

Na mesma direção, Sacristán (1998) define avaliação como sendo:

[...] qualquer processo por meio do qual alguma ou várias características de um aluno/a, de um grupo de estudantes, de um ambiente educativo, de objetivos educativos, de materiais, professores/as, programas etc., recebem a atenção de quem avalia, analisam-se e valorizam-se suas características e condições em função de alguns critérios ou pontos de referência para emitir um julgamento que seja relevante para a educação. (p. 298)

Assim, o processo avaliativo não se resume a apenas 'medir', verificar, mas implica em um julgamento de valor que, por sua vez, implica na tomada de decisões que devem promover melhoria no objeto sob avaliação. Contudo, existem ainda hoje práticas avaliativas baseadas apenas na verificação do rendimento escolar, de modo a cumprir apenas as exigências administrativas da escola. Esteban (2002) considera que tais práticas são realizadas sob a ótica do exame, o que não tem sido muito eficaz no processo de ensino e de aprendizagem, uma vez que não possibilita a observação de um processo tão complexo como esse. 0 problema da avaliação na lógica do exame parece não residir na avaliação em si, mas sim na maneira como ela é encarada. Estabelece-se um padrão que é utilizado como instrumento de comparação, classificação, diferenciação e, sobretudo, de exclusão (2002).

Para que a avaliação possa efetivamente ser utilizada como instrumento de regulação do processo de ensino e aprendizagem de matemática, talvez o primeiro passo seja a

1. No decorrer do trabalho, "Prova" deve ser entendida como a Prova de Questões Abertas de Matemática do AVA/2002. 
mudança na forma como os erros dos alunos são encarados. Pesquisadores da área da Educação têm dado muita atenção aos erros cometidos pelos estudantes (Radatz, 1980; Borasi, 1987; Rico, 1995; Buriasco, 2000; Esteban, 2001; Hadji, 2001; Cury, 2004).

Para Borasi (1987), os erros cometidos pelos estudantes podem, entre outras coisas, ser utilizados como ponto inicial para exploração matemática. De um modo geral, os erros podem ainda ser utilizados como:

a) diagnóstico e remediação: quando o foco da utilização dos erros reside sobre o diagnóstico das causas que levam ao erro e sobre os mecanismos que possam levar à sua superação, de forma que tais causas possam ser evitadas futuramente com outros estudantes; b) investigação: quando os erros são utilizados como mecanismos motivacionais para a investigação sobre o conteúdo matemático relacionado ao erro, à natureza da Matemática e ao próprio processo de ensino e de aprendizagem.

Dessa forma, quando o professor corrige a produção escrita de um estudante e considera apenas a resposta da questão como totalmente certa ou errada, estará perdendo uma oportunidade de verificar os conhecimentos que ele já elaborou e aqueles que estão em processo de elaboração, assim como os erros cometidos pelos estudantes, que podem fornecer importantes informações sobre o processo de ensino e de aprendizagem.

\section{Algumas considerações sobre a educação algébrica}

0 baixo rendimento em Matemática, em particular em relação aos conteúdos algébricos, parece estar relacionado com a maneira como a álgebra é trabalhada nas escolas. Ameron (2002) afirma que, nas escolas em geral, a álgebra escolar tradicional é apresentada aos alunos como um sistema rígido, abstrato e predeterminado, com poucas ligações com o mundo real. Assim, o ensino desta usualmente é iniciado com suas regras sintáticas, com a linguagem simbólica que rapidamente é formalizada, tendo-se o contexto matemático como ponto de partida em detrimento das aplicações dela.

Dentre as inúmeras atividades nas quais os alunos se envolvem durante o processo de ensino e de aprendizagem de álgebra, Ameron (2002) afirma que se podem propor tarefas relacionadas ao pensamento algébrico e à simbolização algébrica com o intuito de que os alunos desenvolvam competências relacionadas ao pensamento e à linguagem algébrica. Analisando os resultados das pesquisas de Vygotsky (2005) sobre pensamento e linguagem, essa ideia pode ficar mais clara. Esse autor, em suas pesquisas, concluiu que a relação existente entre pensamento e palavra é produto do desenvolvimento histórico da consciência humana. Tal relação "não é uma coisa, mas um processo, um movimento contínuo de vaivém do pensamento para a palavra e vice-versa" (p. 156). Assim, conclui-se que pensamento e linguagem são interdependentes, uma vez que

[...] o pensamento nasce através das palavras. Uma palavra desprovida de pensamento é uma coisa morta, e um pensamento não expresso por palavras permanece uma sombra. (p. 190)

Em relação ao pensamento algébrico, Fiorentini, Fernandez e Cristóvão (2005) tomam, como principal referência para identificar seu desenvolvimento, a análise das produções ou resoluções dos estudantes. Para esses autores, o pensamento algébrico pode ser classificado em três fases: a pré-algébrica, a de transição e a do pensamento algébrico mais desenvolvido.

Quando os estudantes iniciam o estudo da álgebra, eles se deparam com algumas dificuldades. Booth (1995) afirma que, para se descobrir por que a álgebra se torna tão difícil para esses estudantes, podem-se identificar os tipos de erros que eles frequentemente cometem e as causas que os levam a cometê-los. 
Tem-se, aqui, que os erros são utilizados com os propósitos de diagnóstico e de remediação (Borasi, 1987), uma vez que a intenção é de se conhecer os mecanismos que estão produzindo esses erros e proporcionar meios para que eles sejam superados.

Assim, se for considerado que um dos objetivos de se trabalhar com álgebra nos EF e EM é fazer com que os alunos desenvolvam o raciocínio algébrico, é fundamental que esses e os demais tipos de erros, quando surgirem, sejam detectados, superados ou aproveitados como oportunidades para explorações matemáticas. Em geral, os erros, bem como os obstáculos e os demais mecanismos produtores destes, podem ser detectados durante o processo avaliativo, desde que este esteja voltado para a aprendizagem do aluno. Assim, num processo avaliativo, cuja perspectiva seja a de contribuir para o processo de ensino e de aprendizagem, a produção escrita do estudante pode fornecer informações sobre a maneira com que ele mobiliza o seu conhecimento para a resolução de problemas.

\section{Procedimentos metodológicos}

Considerando as questões norteadoras e os objetivos desta investigação, optou-se por utilizar uma abordagem predominantemente qualitativa de investigação que, de acordo com Garnica (2004), tem como uma das características

\section{[...] a não neutralidade do pesquisador} que, no processo interpretativo, vale-se de suas perspectivas e filtros vivenciais prévios dos quais não consegue se desvencilhar; além de que a constituição de suas compreensões dá-se não como resultado, mas numa trajetória em que essas mesmas compreensões e também os meios de obtê-las podem ser (re)configuradas. (p. 86)

Por conseguinte, não se pode garantir que os resultados aqui apresentados sejam generalizáveis e não transitórios; não se identificou uma hipótese que se quisesse compro- var ou refutar; na análise interpretativa, as inferências, realizadas durante todo o processo de análise, estão impregnadas das "perspectivas e filtros vivenciais" dos pesquisadores.

A análise da produção escrita dos estudantes referente à Questão ${ }^{2}$ Comum da $8^{\text {a }}$ série do EF e $3^{\text {a }}$ série do EM da AVA/2002 foi realizada à luz da análise de conteúdo (Bardin, 1977), que consiste em um conjunto de técnicas que pretende analisar as formas de comunicação verbal e não verbal.

A Questão Comum é a seguinte: Um encanador A cobra por cada serviço feito um valor fixo de $\mathrm{R} \$ 60,00$ mais $\mathrm{R} \$ 18,00$ por hora de trabalho. Um outro encanador B cobra um valor fixo de $\mathrm{R} \$ 24,00$ mais $\mathrm{R} \$ 36,00$ por hora de trabalho. Sendo t o tempo, medido em horas, para quais valores de t o encanador A fica mais barato que o $B$ ?

Quadro 1: 0 que era esperado dos alunos de acordo com a série.

\begin{tabular}{|c|l|}
\hline Série & \multicolumn{1}{c|}{ Esperado } \\
\hline $\mathbf{8}^{\mathbf{a}} \mathbf{E F}$ & $\begin{array}{l}\text { a) Identifiquem um sistema de equações do primeiro grau que } \\
\text { expressa um problema. } \\
\text { b) Resolvam problema envolvendo um sistema de duas } \\
\text { equações do } 1^{\circ} \text { grau com duas incógnitas. }\end{array}$ \\
\hline $\mathbf{3}^{\mathbf{a}} \mathbf{~ E M}$ & $\begin{array}{l}\text { Identifiquem e resolvam uma inequação do } 1^{\circ} \text { grau que } \\
\text { representa um problema expresso por texto. }\end{array}$ \\
\hline
\end{tabular}

De acordo com Buriasco, Cyrino e Soares (2003), o quadro a seguir expressa o que era esperado dos alunos de cada uma das séries envolvidas ao resolverem essa Questão.

A investigação que deu origem a este artigo baseou-se na análise de 97 Provas $^{3}$ contidas em amostra representativa de alunos do estado do Paraná, sendo 53 Provas de estudantes que em 2002 estavam na $8^{\text {a }}$ série do EF e 44 que estavam na $3^{\text {a }}$ série do EM.

0 primeiro procedimento realizado com as Provas foi uma correção mais geral, de acordo com a proposta do Manual de Correção de

2. Neste trabalho, a expressão Questão deve ser entendida como a Questão Comum à $8^{\mathrm{a}}$ série do ensino fundamental e à $3^{\mathrm{a}}$ série do ensino médio da Prova de Questões Abertas da AVA/2002.

3. Para maiores detalhes sobre como foram selecionadas essas Provas, ver Dalto (2007). 
Questões Abertas (Buriasco; Cyrino; Soares, 2003). Em seguida, procurou-se realizar uma correção mais detalhada com o intuito de verificar não apenas se as resoluções estavam corretas ou não, mas também de verificar as maneiras como os estudantes abordaram a Questão. Em seguida, para facilitar o processo de descrição e de inferência, conforme propõe a análise de conteúdo, as resoluções dos estudantes foram agrupadas de acordo com suas particularidades.

\section{Resultados}

A correção das Provas foi realizada utilizando-se os critérios propostos no Manual para Correção de Provas (Buriasco; Cyrino; Soares, 2003). A proposta desse manual é separar as resoluções dos estudantes em: resolução correta - crédito completo (código 2); resolução parcialmente correta - crédito parcial (código 1); resolução incorreta - nenhum crédito (código 0); sem resolução (em branco) - nenhum crédito (código $9^{4}$ ). Os resultados desse primeiro levantamento quantitativo estão dispostos na Tabela 1.

Observando os dados da Tabela 1 é possível verificar que, basicamente, a produção escrita dos estudantes sugere uma pequena diferença no desempenho destes, quando comparados por série, sendo que é um pouco melhor o desempenho dos da $3^{\text {a }}$ série do EM. Assim, num segundo momento, com o intuito de se verificar a existência de diferenças qualitativas na produção escrita apresentada pelos estudantes nas Provas, foi iniciada uma nova etapa, mais detalhada, de correção. Para a primeira correção, não houve preocupação com o tipo de estratégia apresentada pelo estudante, apenas procurou-se verificar se as resoluções estavam corretas, parcialmente corretas ou incorretas. Já na segunda correção, procurou-se descrever a produção escrita de cada estudante, explicitando a estratégia e o procedimento utilizado ao abordar o problema. Considera-se, neste trabalho, estratégia como a maneira pela qual o estudante abordou o problema. Por exemplo, para resolver um problema, um estudante pode utilizar uma estratégia algébrica, uma aritmética etc. Já o procedimento relaciona-se ao processo de desenvolvimento da estratégia.

Não é uma tarefa fácil classificar o pensamento algébrico do estudante, utilizando-se da sua produção escrita, por pelo menos dois motivos: primeiro, porque se está considerando apenas a sua produção escrita, ou seja, aquilo que ele deixou registrado ao resolver uma questão; em segundo lugar, as categorizações apresentadas na literatura parecem possuir uma zona nebulosa nas linhas divisórias existentes entre as categorias, o que dificulta o trabalho de classificação. Assim, optou-se, neste trabalho, por utilizar apenas duas categorias: algébrica e não algébrica.

$\mathrm{Na}$ Tabela 2, apresentam-se os processos de resolução das questões, independente do crédito que foi atribuído a ela. Considerou-se como não algébrica toda produção escrita na qual existiam apenas operações aritméticas (adição, subtração, multiplicação, divisão) com números. Considerou-se como algébrica a produção escrita que apresentava pelo menos indícios ou da utilização de incógnitas ou variáveis ou da utilização de entes algébricos como equa-

4. No entanto, a amostra de 97 Provas utilizada neste estudo foi determinada de tal forma que não contivesse prova alguma que apresentasse questões em branco, já que o objetivo da investigação era analisar a produção escrita.

Tabela 1: Distribuição das resoluções dos estudantes por crédito obtido e por série.

\begin{tabular}{|c|c|c|c|c|c|c|c|c|}
\hline \multirow{2}{*}{ Série } & \multicolumn{2}{|c|}{$\begin{array}{c}\text { Totalmente correta } \\
\text { (código 2) }\end{array}$} & \multicolumn{2}{|c|}{$\begin{array}{c}\text { Parcialmente Correta } \\
\text { (código 1) }\end{array}$} & \multicolumn{2}{|c|}{$\begin{array}{c}\text { Incorreta } \\
\text { (código 0) }\end{array}$} & \multicolumn{2}{|c|}{ Total } \\
\cline { 2 - 9 } & $\boldsymbol{N}$ & $\%$ & $\boldsymbol{N}$ & $\%$ & $\boldsymbol{N}$ & $\%$ & $\boldsymbol{N}$ & $\%$ \\
\hline $\mathbf{8}^{\text {a }} \mathrm{EF}$ & 7 & 13,2 & 9 & 17,0 & 37 & 69,8 & 53 & 100,0 \\
\hline $\mathbf{3}^{\text {a }} \mathrm{EM}$ & 12 & 27,3 & 10 & 22,7 & 22 & 50,0 & 44 & 100,0 \\
\hline Total da amostra & 19 & 19,6 & 19 & 19,6 & 59 & 60,8 & 97 & 100,0 \\
\hline
\end{tabular}


ções, inequações, funções. Foram considerados como 'outra' a produção escrita que não se enquadrava em nenhuma das classificações anteriores como, por exemplo, aquela que não apresentava qualquer registro de cálculo, mas apresentava uma resposta para o problema.

Tabela 2: Distribuição dos processos de resolução, independente do crédito atribuído à questão.

\begin{tabular}{|c|c|c|c|}
\hline Périe & Nãocesso algébrico & Algébrico & Outro \\
\hline $\mathbf{8}^{\mathbf{a}}-\mathbf{E F}$ & 43 & 06 & 04 \\
\hline $\mathbf{3}^{\mathbf{a}}-\mathbf{E M}$ & 34 & 07 & 03 \\
\hline Total & 77 & 13 & 07 \\
\hline
\end{tabular}

Pode-se verificar, analisando a Tabela 2 e aplicando o Teste Qui-quadrado, excluindo a categoria "outro", que não existe diferença quantitativa significativa $(p=0,5164)$ referente ao processo registrado pelos estudantes em suas produções escritas quando comparados por série, indicando que a escolha do processo de resolução independe da série, ou seja, não se pode afirmar que, por es- tarem em um nível mais elevado de escolaridade, mais estudantes da $3^{\text {a }}$ série do EM escolheram uma estratégia algébrica. Analisando a produção escrita dos estudantes referente à Questão, não se pode afirmar que a maioria dos estudantes não sabe resolver problemas algebricamente, uma vez que o simples fato de não ter escolhido uma estratégia que gerasse uma produção considerada algébrica não revela que o estudante desconheça alguma. 0 que se pode inferir é que a produção escrita dos estudantes revela que eles se sentem mais seguros quando utilizam uma estratégia que gerou uma produção considerada não algébrica.

Após a segunda etapa de correção, procurou-se agrupar as produções escritas da questão sob análise em nove grupos excludentes, uma vez que cada Prova foi classificada em apenas um grupo, conforme critérios apresentados no Quadro 2. Havia Provas que apresentavam características de mais de um grupo. Portanto, para enquadrá-las neste ou naquele grupo, optou-se por utilizar a característica da resolução

Quadro 2: Agrupamento de resoluções de acordo com as estratégias encontradas.

\begin{tabular}{|l|l|c|c|}
\hline \multicolumn{1}{|c|}{ Grupo } & \multicolumn{1}{|c|}{ Critério de agrupamento } & $\begin{array}{c}\mathbf{8}^{\text {a }} \\
\text { série }\end{array}$ & $3^{\text {a }}$ série \\
\hline $\begin{array}{l}\text { G1 } \\
\text { 04 Provas }\end{array}$ & $\begin{array}{l}\text { Calcula o valor do serviço dos encanadores } \\
\text { considerando apenas o valor cobrado por hora. }\end{array}$ & 02 & 02 \\
\hline $\begin{array}{l}\text { G2 } \\
\text { 09 Provas }\end{array}$ & $\begin{array}{l}\text { Subtrai o preço cobrado por hora pelo encanador } \\
\text { B do preço cobrado por hora pelo encanador A. }\end{array}$ & 05 & 04 \\
\hline $\begin{array}{l}\text { G3 } \\
\text { 04 Provas }\end{array}$ & Apenas retira as informações do problema. & 01 & 03 \\
\hline $\begin{array}{l}\text { G4 } \\
\text { 09 Provas }\end{array}$ & $\begin{array}{l}\text { Não apresenta cálculo algum e responde } \\
\text { incorretamente. }\end{array}$ & 06 & 03 \\
\hline $\begin{array}{l}\text { G5 } \\
\text { 12 Provas }\end{array}$ & $\begin{array}{l}\text { Calcula corretamente o valor da primeira hora de } \\
\text { trabalho para ambos os encanadores, } \\
\text { enfatizando a diferença de R\$ 18,00 entre eles. }\end{array}$ & 09 & 03 \\
\hline $\begin{array}{l}\text { G6 } \\
\text { 14 Provas }\end{array}$ & $\begin{array}{l}\text { Calcula o valor da primeira hora ou de alguma } \\
\text { hora especifica de trabalho e responde } \\
\text { incorretamente. }\end{array}$ & 09 & 05 \\
\hline $\begin{array}{l}\text { G7 } \\
\text { 25 Provas }\end{array}$ & $\begin{array}{l}\text { Calcula aritmeticamente o valor das primeiras } \\
\text { horas de trabalho, consecutivas ou não. }\end{array}$ & 11 & 14 \\
\hline $\begin{array}{l}\text { G8 } \\
\text { 10 Provas }\end{array}$ & $\begin{array}{l}\text { Apresenta outros cálculos que não resolvem 0 } \\
\text { problema. }\end{array}$ & 05 & 05 \\
\hline $\begin{array}{l}\text { G9 } \\
\text { 10 Provas }\end{array}$ & $\begin{array}{l}\text { Apresenta as leis das funções que descrevem 0S } \\
\text { custos do serviço dos dois encanadores. }\end{array}$ & 05 & 05 \\
\hline
\end{tabular}


que estivesse diretamente relacionada com a resposta apresentada pelo estudante.

A partir dos agrupamentos feitos, as provas de cada Grupo foram analisadas mais cuidadosamente. Tal análise apresentou indícios de que os estudantes resolvem problemas diferentes daquele problema que foi proposto. Considera-se Problema Proposto aquele que constava originalmente na Prova e que se esperava que fosse resolvido pelo estudante, e Problema Resolvido aquele que, mediante a produção escrita, inferiu-se que cada estudante resolveu como resultado da interpretação que fez do Problema Proposto.

Como exemplo, pode-se citar a produção escrita das Provas do Grupo G1. De modo geral, verifica-se nas Provas o cálculo do valor do serviço dos encanadores, considerando apenas o valor cobrado por hora. Assim, no Problema Resolvido, os estudantes desconsideraram o preço fixo cobrado pelos dois encanadores para serviços de qualquer hora de duração. Tal fato pode ser claramente verificado nas respostas apresentadas nas Provas. Exemplo de resposta
1: "A partir do momento que ao contratarmos o encanador A por horas se tornará mais barato por que ele cobra $\mathrm{R} \$ 18,00$ a hora e o encanador B cobra R\$36,00". Pode-se inferir que o estudante que a escreveu pode ter compreendido que se poderiam contratar os encanadores por hora ou pelo preço fixo. Assim, a resposta apresentada pelo estudante está adequada ao Problema Resolvido, mas não ao Problema Proposto. Exemplo de resposta 2: "O encanador A sempre ficará mais barato se for cobrado só pelo tempo de trabalho". Assim como no exemplo anterior, o estudante que a escreveu pode ter compreendido que os encanadores cobrariam o serviço por um preço fixo, independente do tempo gasto, ou por hora de trabalho. Analisando as respostas apresentadas por estes, pode-se inferir que eles teriam resolvido o seguinte problema, que será denominado Problema Resolvido 1: Um encanador A cobra por cada serviço feito um valor fixo de $R \$ 60,00$ ou $R \$ 18,00$ por hora de trabalho. Um outro encanador $B$ cobra um valor fixo de $R \$ 24,00$ ou $R \$ 36,00$ por hora de trabalho. Em qual das opções o enca-

Quadro 3: Enunciados dos problemas resolvidos inferidos a partir da análise da produção escrita das provas.

Problema Proposto: Um encanador A cobra por cada serviço feito um valor fixo de $R \$ 60,00$ mais $R \$ 18,00$ por hora de trabalho. Um outro encanador B cobra um valor fixo de $R \$ 24,00$ mais $R \$ 36,00$ por hora de trabalho. Sendo " $t$ " $o$ tempo, medido em horas, para quais valores de " $t$ " o encanador $A$ fica mais barato que o $B$ ?

Problema Resolvido 1. Um encanador A cobra por cada senviço feito um valor fixo de $R \$ 60,00$ ou $R \$ 18,00$ por hora de trabalho. Um outro encanador B cobra um valor fixo de $R \$ 24,00$ ou $R \$ 36,00$ por hora de trabalho. Em qual das opções o encanador A é mais barato?

Problema Resolvido 2. Um encanador A cobra, por um serviço de uma hora, um valor fixo de R\$ 60,00 mais $\mathrm{R} \$ 18,00$ e, para serviços com mais de uma hora, um valor de $\mathrm{R} \$ 18,00$ por hora. Um outro encanador B cobra, por um serviço de uma hora, um valor fixo de $\mathrm{R} \$ 24,00$ mais $\mathrm{R} \$ 36,00$ e, para serviços com mais de uma hora, um valor de $\mathrm{R} \$ 36,00$ por hora de trabalho. Em qual das opções o encanador A fica mais barato?

Problema Resolvido 3. Um encanador A cobra por cada serviço feito um valor fixo de $\mathrm{R} \$ 60,00$ mais $\mathrm{R} \$ 18,00$ por hora de trabalho. Um outro encanador B cobra um valor fixo de $R \$ 24,00$ mais $R \$ 36,00$ por hora de trabalho. Sendo $t$ o valor cobrado por hora de trabalho, quantos reais a hora do encanador A é mais barata que do B?

Problema Resolvido 4. Um encanador A cobra por cada serviço feito um valor fixo de $\mathrm{R} \$ 60,00$ mais $\mathrm{R} \$ 18,00$ por hora de trabalho. Um outro encanador B cobra um valor fixo de $R \$ 24,00$ mais $R \$ 36,00$ por hora de trabalho. Sendo t 0 valor cobrado por hora de trabalho, qual valor de t é mais barato?

Problema Resolvido 5. Um encanador A cobra por cada serviço feito um valor fixo de $\mathrm{R} \$ 60,00$ mais $\mathrm{R} \$ 18,00$ por hora de trabalho. Um outro encanador $B$ cobra um valor fixo de $R \$ 24,00$ mais $R \$ 36,00$ por hora de trabalho. Sendo $t$ o tempo, medido em horas, dê um valor de t para o qual o encanador A fica mais barato que o $B$.

Problema Resolvido 6. Um encanador A cobra por cada senviço feito um valor fixo de $\mathrm{R} \$ 60,00$ mais $\mathrm{R} \$ 18,00$ por hora de trabalho. Um outro encanador $B$ cobra um valor fixo de $R \$ 24,00$ mais $R \$ 36,00$ por hora de trabalho. Sendo t o tempo, medido em horas, quantas horas são necessárias para que o encanador A fique mais barato que o B? 
nador A fica mais barato? Pode-se considerar que esse problema e o Problema Proposto referem-se a situações diferentes, mas descritas de maneiras aparentemente semelhantes. É importante destacar que a análise da produção escrita desses estudantes mostra que eles são capazes de resolver problemas como o Problema Resolvido 1, com estratégias e procedimentos que resolveriam também o Problema Proposto.

Analisando as Provas dos demais grupos, foram inferidos outros cinco problemas que supostamente foram resolvidos pelos estudantes, conforme o quadro a seguir.

Após a descrição dos grupos, definiramse quatro categorias a partir das estratégias utilizadas pelos estudantes ao abordarem o problema. A seguir, apresenta-se uma descrição das categorias.

Categoria Custo Não Variável - o custo do serviço de um encanador sempre será menor que o do outro, independente do tempo de duração do serviço - nessa categoria estão as resoluções G1, G5, G6 e G95 ${ }^{5}$, nas quais são utilizados procedimentos algébricos ou não algébricos para encontrar o custo de um serviço de uma duração específica, não obrigatoriamente o custo de um serviço de uma hora. Em parte das Provas dessa categoria, verifica-se a utilização dos valores fixos e dos valores cobrados por hora de ambos os encanadores para encontrar o custo de um serviço. Dessas resoluções, infere-se que os estudantes compreenderam que bastava encontrar o custo de um serviço de uma hora específica de duração e verificar qual dos encanadores teria menor custo para aquele determinado serviço. Em outra parte das Provas dessa categoria, verifica-se a utilização apenas do valor cobrado por hora de trabalho para calcular o valor de um serviço para ambos os encanadores. De acordo com os registros das Provas desse grupo, parece que os estudantes apenas consideraram os valores cobrados por hora de trabalho para calcular os custos dos serviços dos encanadores A e B. Outros parecem ter considerado o valor fixo apenas para um serviço de uma hora de duração.
Categoria Custo Variável - o custo de um dos encanadores não é sempre menor que o custo do outro - nessa categoria, estão as resoluções $\mathrm{G} 7$ e $\mathrm{G} 9$, nas quais são utilizados, por meio de procedimentos algébricos ou não algébricos, os valores fixos e os valores cobrados por hora para calcular o valor de serviços de diferentes horas de duração para ambos os encanadores, consecutivas ou não. Infere-se, a partir da produção escrita dos estudantes presente nas Provas, que desenvolveram uma estratégia que resolve o problema proposto, pois calcularam os custos dos encanadores para serviços de diferentes durações, sendo que a maioria calculou o custo referente a serviços de uma, duas e três horas de duração. Em parte das Provas dessa categoria, verifica-se a utilização de funções para calcular o custo dos encanadores A e B. Infere-se, a partir das resoluções apresentadas nas Provas, que os estudantes desenvolveram uma resolução que se considera, neste trabalho, como uma resolução algébrica do Problema Proposto. Baseando-se na classificação do desenvolvimento do pensamento algébrico de Fiorentini, Fernandez e Cristóvão (2005), pode-se inferir, por meio da análise da produção escrita desses estudantes, que eles se encontram na fase algébrica, uma vez que mesmo não utilizando a linguagem simbólica corretamente, percebe-se que os estudantes foram capazes de escrever as expressões das funções que descreviam os custos dos serviços dos encanadores A e B, o que demonstra algum processo de generalização.

Categoria Diferença - um encanador é mais barato que o outro, dependendo da diferença dos preços cobrados por hora por eles nessa categoria, estão as resoluções $\mathrm{G} 2$, nas quais se subtrai do preço cobrado por hora pelo encanador B o respectivo preço do encanador A. De acordo com a produção escrita registrada nas Provas, pode-se inferir que os estudantes consideraram que se deveria apenas calcular quantos

5. Partes das Provas do Grupo G9 enquadram-se na categoria Custo Não Variável e a outra parte, na categoria Custo Variável. 
reais o valor cobrado por hora do encanador A é mais barato que o respectivo valor cobrado pelo encanador B. Percebe-se, assim como na categoria Custo Não Variável, a utilização equivocada de informações do problema, pois os estudantes não consideraram todas as informações para a solução do problema proposto.

Categoria Outros - nessa categoria, estão outras resoluções $(\mathrm{G} 3, \mathrm{G} 4, \mathrm{G} 8)$ que não solucionam o problema. Essas Provas apresentam estratégias diversas. Em algumas delas, apenas se verifica a resposta; em outras, verificam-se alguns cálculos como já foi descrito.

0 Quadro 4 permite uma melhor compreensão das categorias anteriormente definidas. A Tabela 3 mostra que a grande maioria dos Problemas Resolvidos identificados a partir da produção escrita presente nas Provas apresenta resolução correta. Em 36 das 97 Provas em estudo, foram identificadas resoluções referentes a problemas diferentes daquele que foi proposto. Dessas 36 Provas, 30 delas apre-

Tabela 3: Distribuição das resoluções dos Problemas Resolvidos inferidos na produção escrita dos estudantes em cada série.

\begin{tabular}{|c|c|c|c|c|c|c|}
\hline \multirow{2}{*}{ Resolução } & \multicolumn{3}{|c|}{$8^{\mathrm{a}}$ série } & \multicolumn{3}{c|}{$3^{\mathrm{a}}$ série } \\
\cline { 2 - 8 } Problema & 2 & 1 & 0 & 2 & 1 & 0 \\
\hline Resolvido 1 & 6 & - & - & 2 & - & - \\
\hline Resolvido 2 & 1 & - & - & - & - & - \\
\hline Resolvido 3 & 5 & 1 & - & 3 & - & 2 \\
\hline Resolvido 4 & 1 & - & - & 1 & - & - \\
\hline Resolvido 5 & 3 & - & 1 & 4 & 1 & - \\
\hline Resolvido 6 & 2 & - & - & 2 & - & 1 \\
\hline Total & 18 & 1 & 1 & 12 & 1 & 3 \\
\hline
\end{tabular}

Código 0: resolução incorreta;

Código 1: resolução parcialmente correta;

Código 2: resolução correta

Quadro 4: Categorias definidas a partir das resoluções apresentadas nas Provas.

\begin{tabular}{|c|c|}
\hline Categoria & Descrição \\
\hline $\begin{array}{l}\text { Custo não variável } \\
O \text { custo do serviço de um } \\
\text { encanador sempre será menor } \\
\text { que o do outro, independente } \\
\text { do tempo de duração do } \\
\text { serviço }\end{array}$ & $\begin{array}{l}\text { Nessa categoria, estão as resoluções nas quais são utilizados } \\
\text { procedimentos algébricos ou não algébricos para encontrar o } \\
\text { custo de um serviço de uma duração específica, e não } \\
\text { obrigatoriamente o custo de um serviço de uma hora. Exemplos: } \\
\text { a) verificam-se as operações } 60+18 \bullet 2=96 \text { e } 24+36 \bullet 2=96 \text { e } \\
\text { responde que os dois têm o mesmo preço; } \\
\text { b) verificam-se } 18 \bullet 3=54 \text { e } 36 \bullet 2=72 \text {, respondendo que o } \\
\text { encanador A é mais barato. }\end{array}$ \\
\hline $\begin{array}{l}\text { Custo variável } \\
\text { O custo de um dos } \\
\text { encanadores não é sempre } \\
\text { menor que o do outro }\end{array}$ & $\begin{array}{l}\text { Nessa categoria, estão as resoluções nas quais são utilizados, por } \\
\text { meio de procedimentos algébricos ou não algébricos, os valores } \\
\text { fixos e os valores cobrados por hora para calcular o valor de } \\
\text { serviços de diferentes horas de duração consecutivas ou não para } \\
\text { ambos os encanadores. Exemplo: } \\
\text { Efetua } 24+36 \bullet 1=60 ; 24+36 \bullet 2=96 ; 24+36 \bullet 3=132 ; 60+18 \bullet 1=78 \text {; } \\
60+18 \bullet 2=92 ; 60+18 \bullet 3=114 \text {, respondendo que o encanador A é } \\
\text { mais barato para serviços com duração superior a duas horas. }\end{array}$ \\
\hline $\begin{array}{l}\text { Diferença } \\
\text { Um encanador é mais barato } \\
\text { que o outro, dependendo da } \\
\text { diferença dos preços cobrados } \\
\text { por hora por eles }\end{array}$ & $\begin{array}{l}\text { Nessa categoria, está às resoluções nas quais se subtrai do preço } \\
\text { cobrado por hora pelo encanador B o respectivo preço do } \\
\text { encanador A. } \\
\text { Exemplo: efetua } 36-18=18 \text { e responde que o encanador A é RS } \\
18,00 \text { mais barato que o encanador B. }\end{array}$ \\
\hline Outros & $\begin{array}{l}\text { Nessa categoria, estão outras resoluções que não solucionam o } \\
\text { problema. Exemplos: } \\
\text { a) apenas verifica-se a resposta "o encanador A é mais barato”; } \\
\text { b) apenas efetua } 36 \div 18=2 \text {. }\end{array}$ \\
\hline
\end{tabular}


sentam resolução correta para o Problema Resolvido identificado. Esse resultado sugere que o estudante resolve corretamente o problema que consegue compreender ao ler seu enunciado. Assim, parece que o baixo desempenho dos estudantes não está relacionado ao desconhecimento do instrumental matemático necessário para resolver o problema proposto, como também apontaram as pesquisas de Perego (2005), Nagy-Silva (2005), Segura (2005), Perego (2006), Alves (2006) e Negrão de Lima (2006).

\section{Algumas considerações}

De um modo geral, os resultados deste estudo mostram que menos de $20 \%$ dos estudantes apresentaram uma resolução considerada como correta para a Questão. Entretanto, ainda assim, seria precipitado afirmar que os $80 \%$ restantes "não sabem Matemática". Esse fato fica claro quando são comparadas as Tabelas 1 e 3, já que nessa última percebe-se que os estudantes são capazes de resolver os Problemas Resolvidos inferidos nesta investigação. Dessa forma, em uma avaliação que pretende verificar se os estudantes são capazes de resolver problemas, esse fato não pode ser desconsiderado.

0 baixo desempenho dos estudantes demonstrado (Tabela 1) parece que não é derivado do desconhecimento do instrumental matemático que pode ser utilizado na resolução da Questão, mas que está fortemente relacionado à compreensão do seu enunciado, bem como à identificação do tipo de instrumental mais adequado para resolvê-la. Esse fato ficou claro ao se analisar a produção escrita dos estudantes, principalmente suas respostas, uma vez que muitas delas expressam a diferente compreensão que tiveram do enunciado da Questão.

Verificou-se que a maioria dos estudantes, tanto os da $8^{a}$ série quanto os da $3^{\text {a }}$ série, abordou o problema de uma maneira aqui considerada como não algébrica, envolvendo apenas as operações com números inteiros, usuais nas aulas de matemática da educação básica. É importante destacar que não se pode concluir que esses estudantes desconhecessem alguma estratégia algébrica que resolve a Questão, mas sim que foram capazes de identificar uma estratégia adequada considerada não algébrica. Essa é uma das limitações desta investigação, ou seja, não se pode inferir, a partir da análise da produção escrita dos estudantes referentes à Questão, aquilo que eles não são capazes de fazer. Considerando que os estudantes da $3^{\text {a }}$ série do EM frequentaram a escola por três anos a mais que os estudantes da $8^{\text {a }}$ série do EF, que por conseguinte tiveram acesso a 'mais' conteúdos matemáticos e que a álgebra compreende grande parte de todo o currículo de Matemática, esperava-se que o número de estudantes da $3^{a}$ série que escolheram uma estratégia algébrica fosse maior que o de estudantes da $8^{a}$ série. Portanto, esses resultados levam ao questionamento acerca de como os estudantes estão concebendo o conhecimento algébrico: apenas como uma simples linguagem desprovida de significado sem qualquer aplicabilidade na vida cotidiana? Evidencia-se, portanto, um problema para futuras investigações.

É importante destacar, como era esperado, que se verificou em apenas uma das Provas dos estudantes da $3^{\text {a }}$ série do $\mathrm{EM}$ a resolução da Questão utilizando inequação. Ao aplicar a mesma Questão para um grupo de professores de Matemática, Segura (2005) verificou que apenas 25\% resolveram-na utilizando inequação. Podese considerar, então, que a utilização da inequação como um procedimento de resolução da Questão foi difícil de ser identificado até mesmo para os professores. Uma investigação acerca disso poderia ser desenvolvida para se relacionar tais fatos.

Em um de seus livros, o escritor russo leon Tolstoi (apud Alves, 1993) afirma: "Há quem passe por um bosque e só veja lenha para a fogueira". Essa oração parece se aproximar muito da maneira simplista pela qual o uso que se faz dos resultados da avaliação vem influenciando o modo como os estudantes estão sendo rotulados apenas e simplesmente pelos seus 'fracassos'. No entanto, tomando a avaliação como atividade de 
investigação, este trabalho fornece subsídios que mostram que, enxergando o bosque, os resulta- dos da avaliação podem e devem apontar caminhos a serem percorridos pelos envolvidos na busca de tornarem-se melhores seres humanos para si mesmos e para o mundo.

\section{Referências bibliográficas}

ABRANTES, P. Avaliação e educação matemática. Rio de Janeiro: MEM/USU GEPEM, 1995.

ALVES, J. L. Sabedoria. São Paulo: Gaia, 1993.

ALVES, R. M. F. Estudo da produção escrita de alunos do Ensino Médio em questões de matemática. 2006. 158 p. Dissertação (Mestrado em Ensino de Ciências e Educação Matemática)- Departamento de Matemática, Universidade Estadual de Londrina, Londrina, 2006.

AMERON, B. A. Reinvention of early algebra: developmental research on the transition from arithmetic to algebra [S.I.]: [s.n.], 2002 - Tekst. - Proefschrift Universiteit Utrecht. Disponível em: <http://igitur-archive.library.uu.nl/dissertations/2002-1105161148/inhoud.htm>. Acesso em: 31 ago. 2006.

BARDIN, L. Análise de conteúdo. Tradução de Luís Antero e Augusto Pinheiro. Lisboa: Edições 70, 1977.

B00TH, L. R. Dificuldades das crianças que se iniciam em álgebra. In: COXFORD, A. F.; SHULTE, A. P. (Orgs.). As ideias da álgebra. São Paulo: Atual, 1995. p. 26-36.

BORASI, R. Exploring mathematics through the analysis of errors. For the Learning of Mathematics, v. 7, n. 3, p. 2-8, novembro, 1987.

BURIASCO, R. L. C. Algumas considerações sobre avaliação educacional. Estudos em Avaliação Educacional, São Paulo, n. 22, p. $155-178,2000$.

. Análise da produção escrita: a busca do conhecimento escondido. In: ENDIPE - Encontro Nacional de Didática e Prática de Ensino, XII, 2004, Curitiba. Anais... Curitiba: Champagnat, 2004. v. 3, p. 243-251.

BURIASCO, R. L C. de; CYRINO, M. C. de C. T.; SOARES, M. T. C. Manual para correção das provas com questões abertas de matemática. AVA - 2002. Curitiba: SEED; CAADI, 2003.

CURY, H. N. Análise de erros em educação matemática. Veritati, Salvador, v. 3, n. 4, p. 95-107, 2004.

DALTO, J. 0. A produção escrita em matemática: análise interpretativa da questão discursiva de Matemática comum à $8^{a}$ série do Ensino Fundamental e à $3^{a}$ série do Ensino Médio da AVA/2002. 2007.100p. Dissertação (Mestrado em Ensino de Ciências e Educação Matemática)-Universidade Estadual de Londrina, Londrina. 2007.

ESTEBAN, M. T. (Org.). Avaliação: uma prática em busca de novos sentidos. 3. ed. Rio de Janeiro: DP\&A, 2001.

0 que sabe quem erra? Reflexões sobre avaliação e fracasso escolar. 3. ed. Rio de Janeiro: DP\&A, 2002.

FIORENTINI, D.; FERNANDES, F.; CRISTÓVÃO, E. Um estudo das potencialidades pedagógicas das investigações matemáticas no desenvolvimento do pensamento algébrico. In: SEMINÁRIO LUSO-BRASILEIRO DE INVESTIGAÇÕES MATEMÁTICAS NO CURRÍCULO E NA FORMAÇÃO DO PROFESSOR, 2005, Lisboa. Anais... Lisboa: Faculdade de Ciências da Universidade de Lisboa, 2005. Disponivel em: <http://www.educ.fc.ul.pt/docentes/jponte/seminario_lb.htm>. Acesso em: 13 jan. 2006.

GARNICA, A. V. M. História oral e educação matemática. In: BORBA, M. C.; ARAÚJO, J. L. (Orgs.). Pesquisa qualitativa em educação matemática. Belo Horizonte: Autêntica, 2004. p. 77-98.

HADJI, C. Avaliação desmistificada. Tradução de Patrícia C. Ramos. Porto Alegre: ARTMED, 2001.

NAGY-SILVA, M. C. Do observável para o oculto: um estudo da produção escrita de alunos da $4^{a}$ série em questões de 
matemática. 2005. 123 p. Dissertação (Mestrado em Ensino de Ciências e Educação Matemática)- Departamento de Matemática, Universidade Estadual de Londrina, Londrina, 2005.

NEGRÃO DE LIMA, R. C. Avaliação em matemática: análise da produção escrita de alunos da 4a . série do Ensino Fundamental em questões discursivas. 2006. 201 p. Dissertação (Mestrado em Educação)- Departamento de Educação, Universidade Estadual de Londrina, Londrina, 2006.

PEREGO, F. 0 que a produção escrita pode revelar? Uma análise de questões de matemática. 2006. 127 p. Dissertação (Mestrado em Ensino de Ciências e Educação Matemática)- Departamento de Matemática, Universidade Estadual de Londrina, Londrina, 2006.

PEREGO, S. C. Questões abertas de matemática: um estudo de registros escritos. 2005. 105 p. Dissertação (Mestrado em Ensino de Ciências e Educação Matemática)- Departamento de Matemática, Universidade Estadual de Londrina, Londrina, 2005.

RADATZ, H. Students' errors in the mathematical learning process: a survey. For the Learning of Mathematics, v. 1, n. 1, p. 16 19, 1980.

RICO, L. Errores en el aprendizaje de las matemáticas. In: KILPATRICK, J.; GOMES, P.; RICO, L. Educación matemática. Bogotá: Iberoamericana, 1995. p. 69-108.

SACRISTÁN, J. G. A avaliação no ensino. In: SACRISTÁN, J. G.; PÉREZ GOMES, A. I. Compreender e transformar o ensino. 4. ed. Porto Alegre: Artmed, 1998. p. 295-351.

SEGURA, R. 0. Estudo da produção escrita de professores em questões discursivas de matemática. 2005. 178 p. Dissertação (Mestrado em Educação)- Departamento de Educação, Universidade Estadual de Londrina, Londrina, 2005.

VIGOTSKI, L. S. Pensamento e linguagem. 3. ed. São Paulo: Martins Fontes, 2005.

Recebido em 23.10.08

Aprovado em 10.08.09

Jader Otavio Dalto é acadêmico do doutorado em Ensino de Ciências e Educação Matemática da Universidade Estadual de Londrina e docente da Universidade Federal de Mato Grosso do Sul. E-mail: jader_math@yahoo.com.br.

Regina Luzia Corio de Buriasco é docente do Programa de Pós Graduação em Ensino de Ciências e Educação Matemática da Universidade Estadual de Londrina. 